\title{
Clinical Aspects of Cryptogenic Hepatocellular Carcinoma
}

\author{
KENSHI KOGA \\ The Second Department of Medicine, Kurume University School of Medicine, \\ Kurume 830-0011, Japan
}

\begin{abstract}
Summary: Clinical features of hepatocellular carcinoma in 22 patients without serum HBsAg, HBV DNA, anti-HCV antibody and HCV RNA were characterized and possible pathogenic factors for this cryptogenic hepatocellular carcinoma were prospectively assessed. Twenty-two patients were selected from 434 patients with hepatocellular carcinoma (HCC) who were treated at the Second Department of Internal Medicine, Kurume University Hospital between January 1994 and December 1996. Serum samples collected from these patients were all negative for HBsAg, HBV DNA, anti-HCV antibody, and HCV RNA. Patients were evaluated based on past history, present illness, history of habitual alcohol consumption, results of the serological and biochemical laboratory tests at diagnosis of HCC, Anti-HBc antibody, autoantibodies, GBV-C/HGV RNA, and histopathologic findings of non-cancerous portion of the liver were also evaluated. Among 22 patients with non-B non-C HCC, 16 patients (72.7\%) had a history of alcoholic liver disease, 6 patients had an infection of schistosomiasis Japonica, and 1 patient had Budd-Chiari syndrome. Nine patients (40.9\%) were positive for anti-HBc antibody, but their titers were low in all cases. Among 22 patients, positive for auto antibody, 7 patients (31.8\%) were positive for anti-nuclear antibody, and 17 patients (77.3\%) were positive for anti-smooth muscle antibody. Only 1 patient was positive for GBV-C/HGV RNA. Histopathologic examination was performed in 3 cases for noncancerous portions of the liver. Liver cirrhosis and liver with passive congestive fibrosis were diagnosed in 2 cases each. The remaining one case showed normal feature of the liver. In conclusion, the majority of the 22 patients with non-B non-C HCC had a history of alcoholic liver disease. Many were also positive for auto antibodies. These results suggest that patients with alcoholic liver disease or hepatic disease with autoantibodies may be defined as the high-risk group of developing non-B non-C HCC and should be periodically underwent a complete medical examination.
\end{abstract}

Key words hepatocellular carcinoma (HCC), alcoholic liver disease, autoimmune hepatitis, hepatitis $B$ virus (HBV), hepatitis $C$ virus (HCV)

\section{INTRODUCTION}

The incidence of hepatocellular carcinoma (HCC) in Japan is higher than in western countries [1]. Persistent infection with hepatitis B virus (HBV) or hepatitis $\mathrm{C}$ virus (HCV) is considered as the causative pathogenic factor in $95 \%$ of $\mathrm{HCC}[2,3]$. However, $5 \%$ of patients with HCC are negative for chronic infection of $\mathrm{HBV}$ or $\mathrm{HCV}$ and the clinical characteristics and suspected pathogenic factors in these have not been elucidated.

Although aflatoxines was reported [4-6] as a pathogenic factor other than hepatitis virus, its incidence in Japan was considered low. PCR technique has made it possible to detect $\mathrm{HBV}$ or $\mathrm{HCV}$ infection in a few HCC patients in whom the virus has been previously undetectable. Recently, a new hepatitis virus designated $\mathrm{GB}$ virus $\mathrm{C} /$ hepatitis $\mathrm{G}$ virus (GBV-C/HGV) was isolated [7-9] but it has less importance as a possible causative virus of non 
B non C HCC.

In the present study, we tried to characterize the clinical features specific to HCC patients negative for $\mathrm{HBV}$ or $\mathrm{HCV}$ infection. We tried to assess candidate causative factors involved in the pathogenesis of $\mathrm{HCC}$ in these patients.

\section{PATIENTS AND METHODS}

Twenty-two patients $(5.1 \%)$ were selected from 434 patients with HCC who had been admitted to the Second Department of Medicine, Kurume University Hospital between January 1994 and December 1996. Serum samples from these patients were negative for HBV DNA and HCV RNA.

HCC was diagnosed by histopathologic findings of biopsy specimen aspirated from tumor lesions in the liver, image analysis including ultrasonography, CT, MRI, and angiography. Tests for tumor markers such as $\alpha$-fetoprotein (AFP) and PIVKA-II were also applied. Liver dysfunction was diagnosed by increased AST, ALT, $\gamma$-GTP, and Al-p levels in serum samples. Liver cirrhosis was diagnosed by serological and biochemical laboratory tests, histological findings of the liver biopsy specimens, ultrasonography, CT, and esophageal or gastric varices demonstrated on endoscopy. Patients were interviewed in detail regarding surgical history, past history and present illness, family history of liver disease and history of habitual alcohol consumption. Symptoms before HCC was detected and its tumor stage [10] at diagnosis were also evaluated.

Serum aliquotes were prepared and stored at $-20{ }^{\circ} \mathrm{C}$ until use. Serum samples were tested for antibodies against hepatitis B virus core antigen (anti-HBc antibody), GBV-C/HGV RNA, antinuclear antibody (ANA), anti-smooth muscle antibody (ASMA), and anti-mitochondrial antibody (AMA).

Detection of viral markers for $H B V, H C V$, and $G B V-C / H G V$ in serum samples

HBsAg in serum samples was detected by enzyme immunoassay (MIZUHO MEDY Co., Ltd., Tosu, Japan). Anti-HBc antibody was detected by the RIA method (CORAB ${ }^{\mathrm{TM}}$ kit, Dainabbott Co., Ltd., Tokyo, Japan). When a sample was positive for anti$\mathrm{HBc}$ antibody, the same sample was assayed again at 1:200 dilution to determine antibody. When the diluted sample was still positive for anti-HBc antibody titer ( $\%$ inhibition $>70 \%$ ), the sample was considered to have a high antibody titer; otherwise it was considered to have a low antibody titer. The region of the $\mathrm{S}$ gene of $\mathrm{HBV}$ encoding $\mathrm{HBsAg}$ was amplified by PCR using primers complementary to the 32nd-52nd and to the 272nd-292nd nucleotides.

Anti-HCV was detected by second generation assays (passive hemagglutination assay and/or enzyme immunoassay, Dainabbott Co., Ltd., Tokyo, Japan). HCV RNA was detected by reverse transcriptase "nested" polymerase chain reaction (RT-PCR) using primers derived from the highly conserved 5'-UTR region as described previously [11].

The presence of GBV-C/HGV RNA was detected by RT-hemi-nested PCR. PCR was performed using hemi-nested primers specific for the highly conserved region (5'-UTR) of the GBV-C/HGV gnome as described by Wu et al. [12].

\section{Detection of auto antibodies in serum samples}

ANA, ASMA, and AMA were detected by indirect immunofluorescence test. To detect ANA, serum samples were first mixed with Hep2 cells established from human pharyngeal carcinoma, and then subjected to indirect immunofluorescence test with anti-human immunoglobulins $\operatorname{IgA}, \mathrm{M}$, and $\mathrm{G}$ that were labeled with FITC (fluorescein isothiocyanate) (FLUORO HEPANA TEST', MBL Co., Ltd., Nagoya, Japan). The reaction was defined as positive when either diffuse or granular signals were clearly detected in nuclei. In case of positive results, antibody titration was carried out using samples prepared in a series of repeated two-fold dilutions. To detect ASMA and ANA, drops of serum samples were placed onto sections of stomach (smooth muscle) and kidney specimens from rats fixed on slide glass, respectively. After incubation, antibodies were detected by indirect immunofluorescence test with anti-human IgA, $M$ and $G$ that had been prelabeled with FITC (FLURO AID-1 TEST', MBL Co., Ltd.). When positive signals were detected in the muscular layer of the mucosa, smooth muscles in the muscular layer, submucosal tissue, and vascular wall in the stomach specimen, the serum sample was defined as positive for ASMA. When positive signals were detected in the cytoplasm of distal or proximal renal epidermal cells and in epidermal cells in the gastric wall, the sample was defined as AMA positive. When ASMA and/or AMA were positive, the antibody titration was carried out using samples prepared in a series of repeated two-fold dilutions. 


\section{Statistical analysis}

Statistical analysis was performed by student's ttest. For correlation analysis, Spearman's rank correlation was used. A probability of less than 0.05 was considered significant.

TABLE 1.

Patients profiles in 22 patients with nonB nonC type $H C C$

\begin{tabular}{|c|c|c|c|}
\hline & & Mean $\pm S D$ & range \\
\hline Age & (yrs) & $67.6 \pm 9.1$ & $(47-80)$ \\
\hline \multicolumn{2}{|l|}{ male } & $67.5 \pm 9.3$ & $(47-80)$ \\
\hline \multicolumn{2}{|c|}{ female } & $67.8 \pm 8.7$ & $(51-76)$ \\
\hline T.Bil & $(\mathrm{mg} / \mathrm{dl})$ & $1.3 \pm 0.6$ & $(0.5-3.1)$ \\
\hline AST & (K.U) & $45.2 \pm 21.8$ & $(26-124)$ \\
\hline ALT & (K.U) & $26.3 \pm 17.0$ & $(10-75)$ \\
\hline$\gamma$-GTP & (IU/L) & $127.7 \pm 159.3$ & $(17-797)$ \\
\hline Al-p & (K-A.U) & $12.8 \pm 4.6$ & $(7.4-23.4)$ \\
\hline T.P & $(\mathrm{g} / \mathrm{dl})$ & $7.2 \pm 0.8$ & $(6.0-9.7)$ \\
\hline Alb & $(\mathrm{g} / \mathrm{dl})$ & $3.4 \pm 0.5$ & $(2.5-4.1)$ \\
\hline$\gamma$-glob & $(\mathrm{g} / \mathrm{dl})$ & $1.40 \pm 0.56$ & $(0.72-2.37)$ \\
\hline AFP & (ng/ml) & $69.6 \pm 526.7$ & $(2.8-2074.0)$ \\
\hline PIVKA-II & $(\mathrm{AU} / \mathrm{ml})$ & $6.97 \pm 28.56$ & $(0.063<-137.68)$ \\
\hline
\end{tabular}

\section{RESULTS}

Patients were consisted of 16 males and 6 females with a mean age of $67.6 \pm 9.1$ years (range, 47 to 80 ). Data on serological and biochemical laboratory tests at diagnosis of HCC, and levels for AFP and PIVKA-II are shown in Table 1. Abnormal levels were evident for total bilirubin, AST, $\gamma$-GTP, Al-p, albumin, AFP and PIVKA-II.

As shown in Table 2,8 patients $(36.4 \%)$ had a family history of liver disease, $5(22.7 \%)$ had a history of surgery, and 3 (13.6\%: Pt Nos.5, 7 and 17) had a history of tumor in an organ other than the liver: breast cancer, cervical cancer and double cancers in the colon and stomach, respectively. Three patients $(13.6 \%)$ had a history of blood transfusion.

Regarding habitual alcohol consumption, 16 of 22 patients had alcoholic liver disease with a history of consuming more than $80 \mathrm{~g}$ of ethanol per day for more than 5 years. Thirteen of 16 patients had been consuming more than $140 \mathrm{~g}$ of ethanol per day for more than 10 years.

TABLE 2.

Background of 22 patients with nonB nonC HCC

\begin{tabular}{|c|c|c|c|c|c|c|c|c|c|c|c|}
\hline \multirow{2}{*}{$\begin{array}{l}\text { Pt. } \\
\text { No. }\end{array}$} & \multirow{2}{*}{$\begin{array}{l}\text { Age al } \\
(\operatorname{Sex})\end{array}$} & \multirow{2}{*}{$\begin{array}{l}\text { lcoholic liver } \\
\text { dysfunction }\end{array}$} & \multirow{2}{*}{ SCJ } & \multirow{2}{*}{ anti-HBc } & \multirow{2}{*}{$\begin{array}{l}\text { GBV-C/ } \\
\text { HGV RNA }\end{array}$} & \multirow{2}{*}{ ANA } & \multirow{2}{*}{ ASMA } & \multicolumn{3}{|c|}{ History } & \multirow{2}{*}{ Liver disease } \\
\hline & & & & & & & & LD in family & surgery & transfusion & \\
\hline 1 & $62(\mathrm{M})$ & - & - & N.D. & N.D. & Neg. & Neg. & - & + & - & no dysfunction \\
\hline 2 & $76(\mathrm{~F})$ & - & - & N.D. & N.D. & Neg. & Posi. $\times 20$ & - & - & - & LC \\
\hline 3 & $68(\mathrm{M})$ & - & - & N.D. & N.D. & Neg. & Posi. $\times 40$ & - & - & - & $\mathrm{LC}$ \\
\hline 4 & $63(\mathrm{~F})$ & - & - & N.D. & N.D. & Posi. $\times 80$ & Posi. $\times 40$ & + & - & - & $\mathrm{LC}$ \\
\hline 5 & $73(F)$ & - & - & Posi. & N.D. $\quad \mathrm{P}$ & Posi. X 20 & Posi. $\times 20$ & - & - & - & $\mathrm{CH}$ \\
\hline 6 & $63(\mathrm{~F})$ & - & + & N.D. & N.D. $\quad P$ & Posi. X 80 & Posi. $\times 40$ & - & + & - & $\mathrm{LC}$ \\
\hline 7 & $63(\mathrm{M})$ & + & - & N.D. & N.D. & Neg. & Neg. & + & + & + & $\mathrm{CH}$ \\
\hline 8 & $47(\mathrm{M})$ & + & - & N.D. & N.D. & Neg. & Neg. & - & - & - & $\mathrm{LC}$ \\
\hline 9 & $78(\mathrm{M})$ & + & + & N.D. & N.D. & Neg. & Posi. $\times 20$ & + & - & - & $\mathrm{LC}$ \\
\hline 10 & $63(\mathrm{M})$ & + & - & N.D. & N.D. & Neg. & Posi. $\times 20$ & - & - & - & $\mathrm{LC}$ \\
\hline 11 & $60(\mathrm{M})$ & + & - & N.D. & N.D. & Posi. $\times 160$ & Posi. $\times 20$ & - & - & + & $\mathrm{LC}$ \\
\hline 12 & $61(\mathrm{M})$ & + & + & N.D. & N.D. $\quad \mathrm{P}$ & Posi. $\times 80$ & Posi. $\times 40$ & - & - & - & $\mathrm{LC}$ \\
\hline 13 & $67(\mathrm{M})$ & + & + & N.D. & Posi. & Neg. & Posi. $\times 20$ & + & - & - & $\mathrm{LC}$ \\
\hline 14 & $80(\mathrm{M})$ & + & - & N.D. & N.D. & Neg. & Posi. $\times 20$ & + & - & + & $\mathrm{LC}$ \\
\hline 15 & $57(\mathrm{M})$ & + & - & Posi. & N.D. & Neg. & Neg. & - & - & - & $\mathrm{LC}$ \\
\hline 16 & $56(\mathrm{M})$ & + & - & Posi. & N.D. & Neg. & Posi. $\times 20$ & - & - & - & $\mathrm{LC}$ \\
\hline 17 & $74(\mathrm{~F})$ & + & - & Posi. & N.D. & Neg. & Posi. $\times 40$ & - & + & - & $\mathrm{CH}$ \\
\hline 18 & $76(\mathrm{M})$ & + & + & Posi. & N.D. $\quad P$ & Posi. $\times 80$ & Posi. $\times 40$ & + & - & - & $\mathrm{CH}$ \\
\hline 19 & $51(\mathrm{~F})$ & + & - & Posi. & N.D. & Neg. & Posi. $\times 20$ & + & - & - & $\mathrm{CH}$ \\
\hline 20 & $80(\mathrm{M})$ & + & - & Posi. & N.D. & Neg. & Neg. & - & - & - & $\mathrm{LC}$ \\
\hline 21 & $65(\mathrm{M})$ & + & - & Posi. & N.D. $\quad P$ & Posi. $\times 20$ & Posi. $\times 20$ & + & + & - & $\mathrm{CH}$ \\
\hline 22 & $76(\mathrm{M})$ & + & + & Posi. & N.D. & Neg. & Posi. $\times 20$ & - & - & - & $\mathrm{LC}$ \\
\hline
\end{tabular}

SCJ: schistosomiasis japonica; ANA: anti-nucler antibody; ASMA: anti-smmoth muscle antibody; Posi: positive;

Neg: negative; N.D.: not detected; LC: liver cirrhosis; CH: chronic hepatitis; LD: liver disease 
Five of 6 patients with a history of schistosomiasis Japonica also had a history of alcoholic liver disease. One patient had Budd-Chiari syndrome.

Nine patients $(40.9 \%)$ were positive for anti-HBc antibody, but the titer was low in all cases. Only one patient (Pt No. 13) was positive for GBV-C/HGV RNA.

Seven patients $(31.8 \%)$ were positive for ANA. Of these, 5 had an antibody titration value higher than 80 times dilution. Seventeen patients $(77.3 \%)$ were positive for ASMA positive with a titration value below 40-fold dilution in all cases. All 22 patients were negative for AMA.

Histological examination was performed in 3 patients, using surgically resected liver specimens in 2 patients (Pts No. 1 and 11) and liver biopsy specimen in 1 patient ( $\mathrm{Pt}$ No. 14), of a non-cancerous portions of the liver. In patient 11, histological findings of the resected specimen revealed advanced bridging fibrosis that formed a pseudolobule. Inflammatory cells, mainly lymphocytes and plasmacytes, had infiltrated into the portal areas. This patient had a history of heavy habitual alcohol consumption and was positive for both ANA with a titration value of 160-fold dilution and ASMA with a titration value of 20 -fold dilution, but negative for anti-HBc antibody. However, histological findings of alcoholic liver disease was not observed (Fig. 1). In patient No.14, who had HCC accompanied by BuddChiari syndrome, histological findings of the liver biopsy specimen revealed passive congestive fibrosis. The lesion showed chronic passive congestive

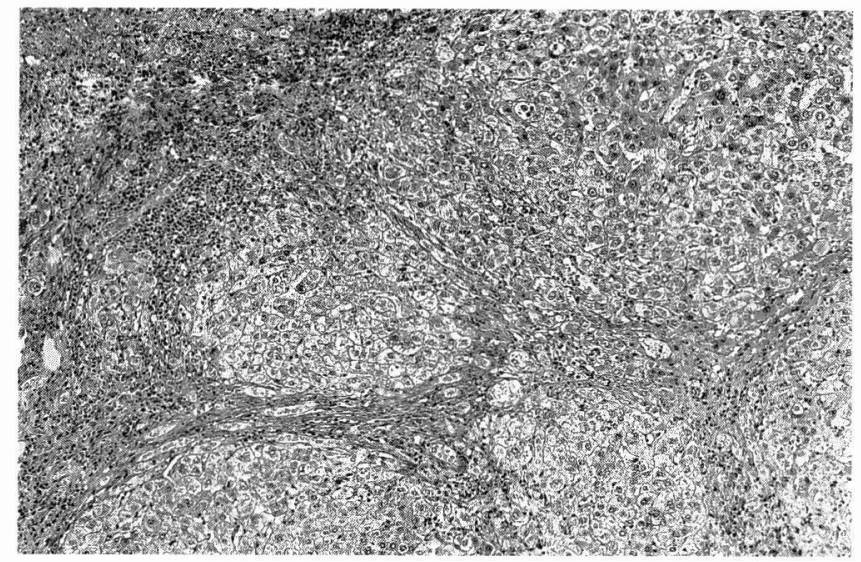

Fig. 1. Histological findings of Pt No. 11 revealed bridging fibrosis conforming a pseudolobule. Inflammatory cells, mainly consisting of lymphocytes and plasmacytes, infiltrated into the portal area. Specific findings of alcoholic liver disease were not detected. $\mathrm{H} \& \mathrm{E}, \times 100$ fibrosis, but neither inflammatory cells infiltrating into the portal areas nor alcoholic liver disease were observed (Fig. 2). In the remaining patient (No. 1), histological findings of resected specimen revealed normal liver (Fig. 3).

In the other 19 patients, 13 had clinically diagnosed as liver cirrhosis and 6 as chronic hepatitis (Table 2).

\section{Symptoms prior to the detection of non- $B$ non- $C$ $\mathrm{HCC}$ and tumor stage at diagnosis}

Seven patients were underwent regular check-up (periodic check-up group) and remaining 15 were not underwent regular check-up (non-periodic check-up group). In 6 patients with liver cirrhosis, HCC was

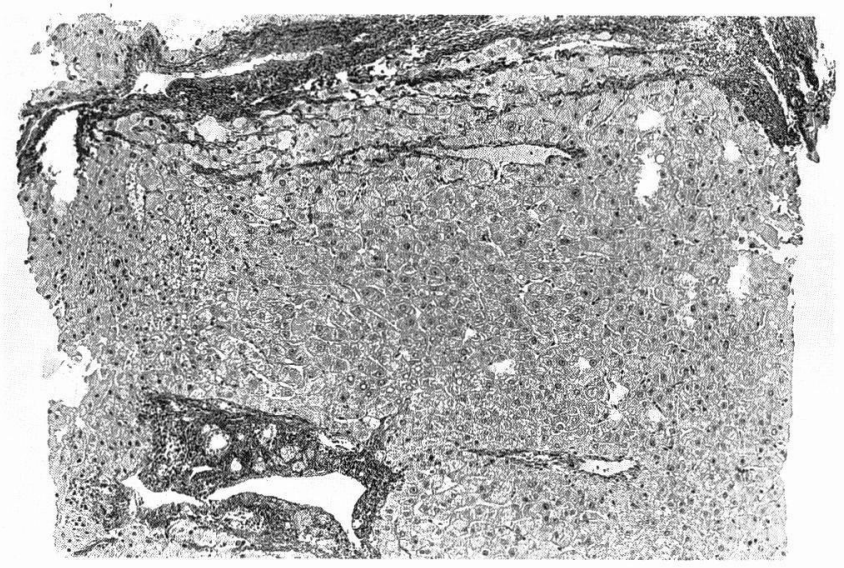

Fig. 2. Histological findings of Pt No. 14 revealed chronic passive congestive fibrosis, but no inflammatory cells infiltrating into the portal area. EGV, $\times 50$

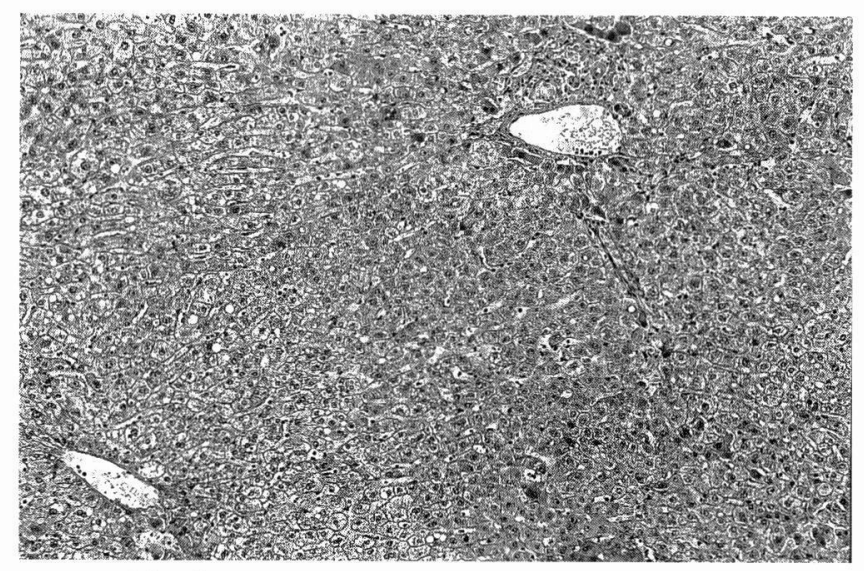

Fig. 3. Histological findings of Pt No. 1 showed no inflammatory cells infiltration or fibrosis. H\&E, $\times 100$ 
detected when they were treated for ascites or bleeding of esophageal varices (Pts No. 4, 10, 13, 15, 16, and 17). HCC was detected in 5 patients when they underwent a regular physical examination (Pts No. 1, 5, 8, 12 and 21). The percentage that HCC was detected in stage I or stage II was $85.7 \%(6 / 7)$ in the periodic check-up group and $40 \%(6 / 15)$ in the non-periodic check-up group, respectively. Thus, the incidence of HCC detected in stage I or stage II was significantly higher in the periodic check-up group than that of non-periodic check-up group $(\mathrm{p}<0.05)$.

\section{DISCUSSION}

In assessing causative factors for development of HCC in 205 patients, Shiratori et al. reported $4.4 \%$ of the cases were not associated with HBV or HCV infection [2], consisting to the findings in the present study. Characterization of clinical features specific to this cryptogenic $\mathrm{HCC}$ and the search for candidate causative factors involved in development HCC is obviously important to add a new category of patients at high-risk of developing non-B non-C HCC. These findings are also crucial to understanding the underlying mechanism of carcinogenesis in cryptogenic HCC. Among 22 patients with HCC evaluated in the present study, as many as 16 had a history of alcoholic liver disease. Five had schistosomiasis Japonica or Budd-Chiari syndrome as an accompanying disease. Although ethanol is a potential carcinogenic promoter [13], the incidence of HCC in which heavy habitual consumption of alcohol alone may have caused the liver disease is low in Japanese [14]. However, it should be emphasized that habitual consumption of alcohol is a well known risk factor for liver cirrhosis $[15,16]$, and HCC often develops in patients with liver cirrhosis. Therefore, alcoholic liver disease by itself could be a carcinogenic condition promoting HCC. Eight of 16 patients $(50 \%)$ with alcoholic liver disease were positive for anti-HBc antibody though negative for HBV DNA. This positivity rate for anti$\mathrm{HBc}$ antibody was not very high compared with that generally seen in the local population [17]. However, more sensitive methods of detecting HBV DNA in serum and liver tissue may demonstrate a higher HBV positivity rate and provide a clue for a possible relationship between $\mathrm{HBV}$ and pathogenesis of this cryptogenic HCC.

GBV-C/HGV RNA was detected only in 1 of the 22 patients. This low incidence is consistent with the reported by Sugai et al. [18] that GBV-C/HGV RNA was detected only in 1 of 12 patients with non-B non-C chronic liver disease. Kao et al. [19] also reported co-infection with $\mathrm{GBV}-\mathrm{C} / \mathrm{HGV}$ in patients who were positive for hepatitis $\mathrm{B}$ or hepatitis $\mathrm{C}$ did not cause any alteration in clinical features during follow-up with respect to development of HCC. Taken together with the present findings, involvement of GBV-C/HGV in the pathogenesis of non-B non-C HCC may be unlikely.

The high incidence of schistosomiasis Japonica in the present cases $(6 / 22)$ is likely due to the fact this disease was previously endemic in this area [20]. In our previous study, HBV or HCV infection was detected in serum samples from all 25 patients with schistosomiasis Japonica who developed HCC [21]. These results strongly suggest that infection with HBV or HCV plays an important role in the pathogenesis of HCC in patients regardless of a history of schistosomiasis Japonica.

Several reports suggest that patients with BuddChiari syndrome are predisposed to HCC [22-24]. The only one patient with Budd-Chiari syndrome in the present case (Pt No. 14) was negative for all known hepatitis viral markers and did not show any particular histological anomaly in the non-cancerous portion of the liver beyond the ordinary chronic passive congestive fibrosis seen in alcoholic liver disease. In this patient, therefore, chronic congestion in the liver may have caused HCC.

Rayder et al. [4] reported that HCV infection was often detected in patients with autoimmune hepatitis (AIH) who developed HCC. Komada et al. [5] reported that a patient with primary biliary cirrhosis (PBC) accompanied by HCC was negative for all $\mathrm{HBV}$ related markers, anti-HCV antibody, and HCV RNA in serum. In our patient 11 (Table 2), autoimmune hepatitis was also implicated by the finding of inflammatory cells, primarily lymphocytes and plasmacytes, had infiltrated the portal areas. These results suggest that there may be a causal relationship between autoimmune hepatitis and the development of HCC, although the incidence is low. This issue deserves further investigation.

Regarding the incidence of patients positive for autoantibody in this study, the positivity rate for ANA and ASMA was as high as $30.8 \%$ (7/22) and $77.3 \%$ (17/22), respectively. The positivity rate in patients with HCC has been reported to be significantly higher than that in patients with chronic hepatitis, those with liver cirrhosis, or with tumor in organs other than the liver [25]. There is also a report that the development of HCC was accompanied by a 
conversion from negative to positive ANA with an antibody titer in increments [26]. Thus, the high incidence of ANA positivity among HCC patients may reflect malignant transformation of hepatocytes. The high incidence of ANA positivity in the present cases may be explained by this carcinogenic event.

Among 7 patients who periodically underwent a complete medical examination for alcoholic liver disease or cryptogenic liver disorders that are prone to development of HCC, HCC was detected in 6 patients in its earlier stage (stage I or II). The tumor stage when HCC was detected among patients in the periodic follow-up group was earlier than that detected among those who did not undergo the periodic follow-up.

The clinical aspects of the non-B non-C cryptogenic HCC studied here suggest that patients with alcoholic liver disease, autoimmune hepatitis, or auto antibody should also undergo follow-ups for HCC. The present findings warrant further research to identify other viruses that are carcinogenic to the liver.

ACKNOWLEDGMENTS: The author thanks Prof. Kyuichi Tanikawa, Associate Prof. Michio Sata, Dr. Hiroshi Suzuki and Dr. Naofumi Ono (The Second Department of Medicine, Kurume University School of Medicine) for clinical advice and suggestions. The author also thanks Prof. Masamichi Kojiro and Associate Prof. Masayoshi Kage (The First Department of Pathology, Kurume University School of Medicine) for pathological advice.

\section{REFERENCES}

1. Parkin DM, and Muir CS. Cancer incidence in five continents. Comparability and quality of data. IARC Sci Publ 1992; 120:45-173.

2. Siratori $Y$, Shiina S, Imamura M, Kato N, Kanai F et al. Characteristic difference of hepatocellular carcinoma between hepatitis B- and C-viral infection in Japan. Hepatology 1995; 22:1027-1033.

3. Tokesi S, Sata M, and Tanikawa K. Detection of serum HBV DNA and anti-HCV antibody in HBsAg seronegative patients with hepatocellular carcinoma in Japan. The Japanese Association for Infectious Disease 1995; 22:103-107.

4. Ryder SD, Koskinas J, Rizzi P, McFarlane IG, Portmann $\mathrm{BC}$ et al. Hepatocellular carcinoma complicating autoimmune hepatitis: Role of hepatitis $\mathrm{C}$ virus. Hepatology 1995; 22:718-722.

5. Komada N, Yamagata M, Komura K, Hayasi K, Maruyama $\mathrm{Y}$ et al. Hepatocellular carcinoma with sarcomatous change arising in primary biliary cirrhosis. J Gastroenterol 1997; 32:95-101.

6. Takayasu K, Muramatsu Y, Moriyama N, Wakao F, Makuuchi $\mathrm{M}$ et al. Radiological study of idiopathic
Budd-Chiari complicated by hepatocellular carcinoma a report of four cases. Am J Gastroenterol 1994; 89:249253.

7. Simons JN, Leary TP, Dawson GJ, Pilot-Matias TJ, Muerhoff AS et al. Isolation of novel virus-like sequences associated with human hepatitis. Nat Med 1995; 1:564-569.

8. Leary TP, Muerhoff AS, Simmons JN, Pilot-Masias TJ, Erker JC et al. Seaquences and genomic organization of GBV-C: A novel member of the flaviviridae associated with human non-A-E hepatitis. J Med Virol 1996; 48:6067.

9. Linnen J, Wages J Jr, Zhang Keck ZY, Fry KE, Krawczynski $\mathrm{KZ}$ et al. Molecular cloning and disease association of hepatitis $\mathrm{G}$ virus: a transufusiontranamissible agent. Science 1996; 271:505-508.

10. Hermanek $P$, and Sobin LH. TNM classification of malignant tumors. 4th edition, UICC, Springer Verlag, Berlin, 1987.

11. Okamoto H, Okada S, Sugiyama Y, Tanaka T, Sugai T et al. Detection of hepatitis C virus RNA by a two-stage polymerase chain reaction with two pairs of primers deduced from the 5'-nonconding region. Jpn J Exp Med 1996; 60:215-222.

12. Wu RR, Mizokami M, Cao K, Nakano T, Ge XM et al. GB virus C/Hepatitis $G$ virus infection in Southern China. J Infect Dis 1997; 175:168-171.

13. Seitz HK. Ethnol and carcinogenesis. In: Gastroenterology. ed. Konnerrell B, Sprringer-Verlag, Berlin, Heidelberg, pp192-212, 1985.

14. Sata M, Fukuizumi K, Uchimura $Y$, Nakano H, Ishi K et al. Hepatitis $\mathrm{C}$ virus infection in patients with clinically diagnosed alcoholic liver disease. Journal of Viral Hepatitis 1996; 3:143-148.

15. Corrao G, Arico S, Zambon A, Torchio P, Lepore AR et al. Is alcohol a risk factor for liver cirrhosis in HBsAg and anti-HCV negative subjects. J Hepatol 1997; 27:470476.

16. Hisatomi S, Kumashiro R, Sata M, Ishi K, and Tanikawa K. Gender difference in alcoholic liver disease in Japan an analysis based on histological findings. Hepatology Research 1997; 8:113-120.

17. Noguchi S, Sata M, Suzuki H, Mizokami M, and Tanikawa K. Routs of tranaimission of hepatitis $\mathrm{C}$ virus in an endemic rural area of Japan. Scand J Infect Dis 1997; 29:23-28.

18. Sugai Y, Nakayama H, Fukuda M, Sawada N, Tanaka T et al. Infection with $\mathrm{GB}$ virus $\mathrm{C}$ in patients with chronic liver disease. J Med Virol 1997; 51:175-181.

19. Kao JH, Chen PJ, Lai MY, Chen W, Liu DP et al. GB virus $C$ /hepatitis $G$ virus infection in an area endemic for viral hepatitis, chronic liver disease, and liver cancer. Gastroenterology 1997; 112:1265-1270.

20. Kitani K, and Iuchi S. Schistosomiasis japonica: a vanishing endemic in Japan. J Gastroenterol Hepatol 1990; 5:160-172.

21. Uchimura Y, Sata M, Suzuki H, Akiyosi F, Tanaka K et al. High prevalence of hepatitis $\mathrm{C}$ virus infection in Schistosomiasis japonica patients associated with hepatocellular carcinoma. Int J Oncol 1997. (in press) 
22. Okuda H, Yamagata H, Obata H, Iwata H, Sasaki R et al. Epidemiological and clinical features of Budd-Chiari syndrome in Japan. J Hepatology 1993; 22:1-9.

23. Kage $M$, Arakawa $M$, Kojiro $M$, and Okuda $K$. Histopathology of membranous obstruction of the inferior vena cava in the Budd-Chiari syndrome. Gastroenterology 1992; 102:2081-2090.

24. Kew MC, McKnight A, Hodkinson J, Bukofzer S, and Esser JD. The role of membranous obstruction of the inferior vena cava in the etiology of hepatocellular carcinoma in South African Blacks. Hepatology 1992; 9:121-125.

25. Imai H, Ochs RL, Kiyosawa K, Furuta S, Nakamura RM et al. Nucleolar antigens and auto antibodies in hepatocellular carcinoma and other malignancies. Am J Pathol 1992; 140:859-870.

26. Imai $H$, Nakano $H$, Kiyosawa $K$, and Tan EM. Increasing titers and changing specificities of antinuclear antibodies in patients with chronic liver disease who develop hepatocellular carcinoma. Cancer 1992; 71:26-35. 